\title{
Periodic solutions of stochastic coupled systems on networks with periodic coefficients
}

\author{
Jichao Wang ${ }^{\mathrm{a}}$, Xinhong Zhang ${ }^{\mathrm{a}}$, Wenxue $\mathrm{Li}^{\mathrm{b}, *}$ \\ ${ }^{a}$ College of Science, China University of Petroleum (East China), Qingdao 266580, PR China \\ ${ }^{b}$ Department of Mathematics, Harbin Institute of Technology(Weihai), Weihai 264209, PR China
}

\begin{abstract}
This paper is concerned with periodic solutions of periodic stochastic coupled systems on networks. A systematic method of proving the existence of periodic solutions to the general stochastic coupled systems on networks is provided by using combined method of graph theory and Lyapunov method. Moreover, sufficient conditions for the existence of the periodic solutions to a type of stochastic coupled system on networks are established. In addition, based on Lyapunov method and graph theory, global asymptotic stability criterion for the periodic solution is also given. Finally, a numerical example is provided to illustrate the effectiveness of the results developed.
\end{abstract}

Keywords: periodic solution; stochastic coupled systems; graph theory; Lyapunov method

\section{Introduction}

In recent years, the theory of stochastic differential equations (SDEs) has attracted much attention since it is of practical importance, and has an important role in many ways such as in insurance, finance, population dynamics, control etc. [1]. There are many good books and papers which study SDEs and we here mention Arnold [2], Friedman [3], Has'minskii [4] and Mao [5, 6] among others. Stability of SDEs has been well studied. For example, Mao [5] discussed several kinds of stochastic stabilities of SDEs and stochastic functional differential equations (SFDEs). Most of the existing papers on the stability of SDEs and SFDEs are mainly concerned with the stability of the equilibrium point. However, in the real world, most systems are in the periodic environment, and periodicity of dynamical systems is an interesting topic. But the existence and stability of periodic solution to the periodic stochastic differential equations are not well studied. Up to now, only a few papers and books about the periodic stochastic differential equations have been reported $[4,7,8,9,10]$. In [4], Hasminskii gave some basic results on the periodic solutions of SDEs. Using these results in [4], Zhang and Gopalsamy [7] studied two $n$-species stochastic population models with periodic coefficients. In papers [8, 9], the authors studied the following periodic stochastic differential equation with finite delay or infinite delay

$$
\mathrm{d} x(t)=f\left(t, x_{t}\right) \mathrm{d} t+g\left(t, x_{t}\right) \mathrm{d} B(t)
$$

and gave some sufficient conditions for the existence of periodic solutions.

\footnotetext{
${ }^{*}$ Corresponding author.

Email address: wenxue810823@163.com (Wenxue Li)
} 
On the other hand, coupled systems on networks (CSNs) have been an active research area due to their applications in the fields such as artificial complex dynamical systems, neural networks, the spread of infectious diseases, biological fields and so on (see $[11,12,13,14,15,16,17]$ and references therein). The dynamic properties, especially the stability, of CSNs have been widely studied in recent years, and many research papers have been reported (see Refs. [18, 19, 20, 23, 24, 26, 27, 28, 29]). In paper [18], by using the results of graph theory, Li and Shuai successfully developed a systematic method to obtain the global asymptotical stability of CSNs. Paper [20] studied the global stability for stochastic coupled systems on networks (SCSNs). By using the method in [18], combining coincidence degree theory, Zhang and Li studied the existence and global exponential stability of the periodic solution to CSNs with time delay and neutral CSNs with delays [21, 22]. The above papers $[18,21,22]$ investigated the global stability of equilibria or the existence and global stability of the periodic solution to CSNs by using graph theory. As far as we know, the existence and global stability of the periodic solution to periodic stochastic coupled systems on networks have been rarely studied.

Motivated by above facts, in this paper, we mainly discuss the periodic solutions of SCSNs and establish a theoretical framework for the existence of periodic solutions to SCSNs. The major research methods are graph theory and Lyapunov method. As applications, we establish the sufficient principle for the existence and global asymptotic stability of periodic solution to a type of stochastic coupled system on networks. Finally, a numerical example is provided to illustrate the effectiveness of the theoretical results.

\section{Main lemmas}

For the sake of simplicity, we use the following notations in the sequel. Let $\mathbb{R}^{+}=[0, \infty)$ and $\mathbb{L}=\{1,2, \cdots, l\}$. Denote by $|\cdot|$ the Euclidean norm for vectors. $C^{2,1}\left(\mathbb{R}^{n} \times \mathbb{R}^{+} ; \mathbb{R}^{+}\right)$denotes the space of all nonnegative functions which are twice continuously differentiable with respect to $x$ and once continuously differentiable with respect to $t .\left(\Omega, \mathcal{F},\left\{\mathcal{F}_{t}\right\}_{t \geq 0}, \mathbb{P}\right)$ is a complete probability space with a filtration $\left\{\mathcal{F}_{t}\right\}_{t \geq 0}$ satisfying the usual conditions. If $f(t)$ is a continuous bounded function on $[0, \infty)$, define

$$
f^{u}=\sup _{t \in[0, \infty)} f(t), \quad f^{l}=\inf _{t \in[0, \infty)} f(t) .
$$

\subsection{Graph theoretical results}

In this paper, we will use the following basic lemma on graph theory. The concepts concerning graph theory are given in Appendix.

Lemma 1. [18] Assume $l \geq 2$. Let $c_{k}$ denote the cofactor of the $k$-th diagonal element of Laplacian matrix of $(G, A)$. Then the following identity holds:

$$
\sum_{k, h=1}^{l} c_{k} a_{k h} F_{k h}\left(y_{k}, y_{h}\right)=\sum_{Q \in \mathbb{Q}} W(Q) \sum_{(s, r) \in E\left(C_{Q}\right)} F_{r s}\left(y_{r}, y_{s}\right) .
$$

Here $F_{r s}\left(y_{r}, y_{s}\right), 1 \leq r, s \leq l$, are arbitrary functions, $\mathbb{Q}$ is the set of all spanning unicyclic graphs of $(\mathcal{G}, A), W(Q)$ is the weight of $Q$, and $C_{Q}$ denotes the directed cycle of $Q$. In particular, if $(G, A)$ is strongly connected, then $c_{k}>0$ for $k=1,2, \cdots, l$. 


\subsection{Periodic stochastic process}

Lyapunov method plays an important role in the study of the existence of periodic solutions of SDEs. The following definitions and lemmas are taken from $[4,9]$.

Definition 1. A stochastic process $x(t)$ is said to be periodic with period $T$ if its finite dimensional distributions are periodic with period $T$, i.e., for any positive integer $m$ and any moments of time $t_{1}, \cdots, t_{m}$, the joint distributions of the random variables $x_{t_{1}+k T}, \cdots, x_{t_{m}+k T}$ are independent of $k(k= \pm 1, \pm 2, \cdots)$.

Lemma 2. Consider the following periodic SDE

$$
\mathrm{d} x(t)=b(x(t), t) \mathrm{d} t+\sigma(x(t), t) \mathrm{d} B(t),
$$

where the coefficients $b: \mathbb{R}^{n} \times \mathbb{R}^{+} \rightarrow \mathbb{R}^{n}, \sigma: \mathbb{R}^{n} \times \mathbb{R}^{+} \rightarrow \mathbb{R}^{n \times m}, b(x, t+T)=b(x, t), \sigma(x, t+T)=\sigma(x, t)$ for some $T>0$, and $B(t)$ is an m-dimensional Browian motion. Suppose that system (1) has the global solutions. Let $V(x, t) \in C^{2,1}\left(\mathbb{R}^{n} \times \mathbb{R}^{+} ; \mathbb{R}^{+}\right)$which is $T$-periodic in $t$, and satisfy the conditions

$$
\begin{gathered}
\inf _{\|x\|>l} V(x, t) \rightarrow \infty \text { as } l \rightarrow \infty, \\
\inf _{\|x\|>l} L V(x, t) \rightarrow-\infty \text { as } l \rightarrow \infty .
\end{gathered}
$$

Then there exists a solution of (1) which is a T-periodic markov process, where

$$
L V(x, t)=\left[\frac{\partial V}{\partial t}+\frac{\partial V}{\partial x} b+\frac{1}{2} \operatorname{trace}\left(\sigma^{\mathrm{T}} \mathrm{V}_{\mathrm{xx}} \sigma\right)\right](x, t),
$$

where

$$
\frac{\partial V}{\partial x}=\left(\frac{\partial V}{\partial x_{1}}, \cdots, \frac{\partial V}{\partial x_{n}}\right), \quad V_{x x}=\left(\frac{\partial^{2} V}{\partial x_{i} \partial x_{j}}\right)_{n \times n} .
$$

For Eq.(1), we give several kinds of stochastic stabilities which can be found in [30].

Definition 2. (i). The solution $x(t)=0$ is said to be stochastically stable if for every $\varepsilon>0$ and $s \geq t_{0}$

$$
\lim _{x_{0} \rightarrow 0} \mathbb{P}\left(\sup _{[s, \infty)}\left\|x\left(t ; s, x_{0}\right)\right\| \geq \varepsilon\right)=0 .
$$

(ii). $x(t)=0$ is stochastically asymptotically stable if, in addition,

$$
\lim _{x_{0} \rightarrow 0} \mathbb{P}\left(\lim _{t \rightarrow \infty}\left\|x\left(t ; s, x_{0}\right)\right\|=0\right)=1, s \geq t_{0} .
$$

(iii). $x(t)=0$ is said to be globally asymptotically stable if, further,

$$
\mathbb{P}\left(\lim _{t \rightarrow \infty} x\left(t ; s, x_{0}\right)=0\right)=1, \forall x_{0} \in \mathbb{R}^{n}
$$




\section{The existence of periodic solutions of stochastic coupled systems on networks}

Based on graph theory, coupled systems can be described in a digraph. Now we demonstrate the construction of a stochastic coupled system on networks on a digraph $\mathcal{G}$ with $l(l \geq 2)$ vertices. Assume that each subsystem dynamics is described by

$$
\mathrm{d} x_{k}(t)=f_{k}\left(t, x_{k}(t)\right) \mathrm{d} t+g_{k}\left(t, x_{k}(t)\right) \mathrm{d} B_{k}(t),
$$

where $x_{k}(t)=\left(x_{k 1}(t), x_{k 2}(t), \cdots, x_{k m}(t)\right)^{T}$ represents the state of the $k$-th subsystem, the $T$-periodic functions $f_{k}$, $g_{k}: \mathbb{R}^{+} \times \mathbb{R}^{m} \rightarrow \mathbb{R}^{m}$ are the drift coefficient and the diffusion coefficient, respectively, $B_{k}(t)$ is one-dimensional Browian motion defined on $\left(\Omega, \mathcal{F},\left\{\mathcal{F}_{t}\right\}_{t \geq 0}, \mathbb{P}\right)$. We assume that the influence of the $h$-th subsystem on the $k$-th subsystem for the drift coefficient and the diffusion coefficient are described by $M_{k h}\left(t, x_{h}(t)\right)$ and $N_{k h}\left(t, x_{h}(t)\right)$, respectively. Here $M_{k k}=N_{k k}=0$ and $M_{k h}=N_{k h}=0$ if and only if there exists no influence from $h$-th subsystem to $k$-th subsystem. Then we can obtain an $m$-dimensional stochastic coupled system as follows:

$$
\mathrm{d} x_{k}(t)=\left[f_{k}\left(t, x_{k}(t)\right)+\sum_{h=1}^{l} M_{k h}\left(t, x_{h}(t)\right)\right] \mathrm{d} t+g_{k}\left(t, x_{k}(t)\right) \mathrm{d} B_{k}(t)+\sum_{h=1}^{l} N_{k h}\left(t, x_{h}(t)\right) \mathrm{d} B_{h}(t), k \in \mathbb{L} .
$$

We assume that the coefficients of (4) are $T$-periodic in $t$. Stochastic coupled system (4) is constructed based on a digraph $\mathcal{G}$, in which every node of $\mathcal{G}$ represents a subsystem and the weight of arc $(h, k)$ represents the influence of the $h$-th subsystem on the $k$-th subsystem. For example, for a digraph $\mathcal{G}$ with 4 vertices, the network among compartments can be depicted in Figure 1.

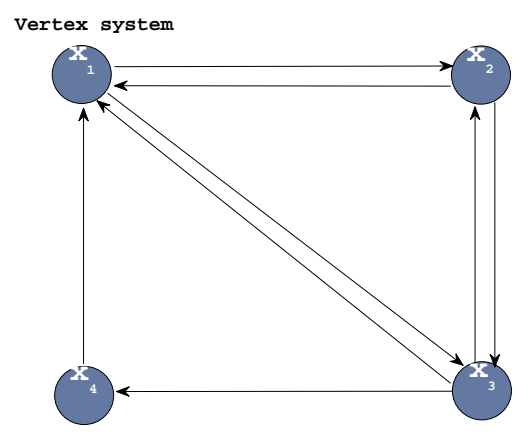

Figure 1: The structure of the coupled system.

For the aim of this paper, we assume that the functions $f_{k}, M_{k h}, g_{k}$ and $N_{k h}$ are such that system (4) has a unique global solution. In the following theorem, we will develop a systematic approach to obtain the existence of periodic solutions of system (4) by using graph theory and Lyapunov method.

Theorem 1. Assume that the following conditions hold:

(V1) There are some positive constants $p \geq 2, a_{k}$, $b_{k}$ and function $V_{k}\left(x_{k}, t\right) \in C^{2,1}\left(\mathbb{R}^{m} \times \mathbb{R}^{+} ; \mathbb{R}^{+}\right)$such that

$$
a_{k}\left|x_{k}\right|^{p} \leq V_{k}\left(x_{k}, t\right) \leq b_{k}\left|x_{k}\right|^{p} .
$$


(V2) There are constants $\sigma_{k}>0, \gamma_{k} \geq 0$ and $a_{k h} \geq 0$ such that

$$
L V_{k}\left(x_{k}(t), t\right) \leq-\sigma_{k}\left|x_{k}(t)\right|^{p}+\sum_{h=1}^{l} a_{k h} F_{k h}\left(x_{k}(t), x_{h}(t)\right)+\gamma_{k} .
$$

(V3) Digraph $(\mathcal{G}, A)\left(A=\left(a_{k h}\right)_{l \times l}\right)$ is strongly connected and along each directed cycle $C$ of weighted digraph $(\mathcal{G}, A)$, there is

$$
\sum_{(h, k) \in E(C)} F_{k h}\left(x_{k}, x_{h}\right) \leq 0
$$

for all $x_{k}, x_{h} \in \mathbb{R}^{m}$.

Then system (4) has a T-periodic solution.

Proof. Let $V(x, t)=\sum_{k=1}^{l} c_{k} V_{k}\left(x_{k}, t\right)$, where $c_{k}$ denotes the cofactor of the $k$-th diagonal element of Laplacian matrix of $\left(\mathcal{G},\left(a_{k h}\right)_{l \times l}\right)$, and from the strong connectedness of $(\mathcal{G}, A)$ it follows that $c_{k}>0$. Then from condition (V1) it follows that

$$
V(x, t)=\sum_{k=1}^{l} c_{k} V_{k}\left(x_{k}, t\right) \leq \sum_{k=1}^{l} c_{k} b_{k}\left|x_{k}\right|^{p} \leq l^{\frac{p}{2}}\left(\sum_{k=1}^{l} c_{k} b_{k}\right)|x|^{p}=: b|x|^{p},
$$

and

$$
\begin{aligned}
& \sum_{k=1}^{l} c_{k} a_{k}\left|x_{k}\right|^{p} \\
= & \sum_{i=1}^{l} c_{i} a_{i} \sum_{k=1}^{l}\left[\frac{c_{k} a_{k}}{\sum_{j=1}^{l} c_{j} a_{j}}\left(\left|x_{k}\right|^{2}\right)^{\frac{p}{2}}\right] \\
\geq & \sum_{i=1}^{l} c_{i} a_{i}\left[\sum_{k=1}^{l} \frac{c_{k} a_{k}}{\sum_{j=1}^{l} c_{j} a_{j}}\left|x_{k}\right|^{2}\right]^{\frac{p}{2}} \\
\geq & \left(\sum_{k=1}^{l} c_{k} a_{k}\right)^{1-\frac{p}{2}}\left(\min _{k \in \mathbb{L}}\left\{c_{k} a_{k}\right\}\right)^{\frac{p}{2}}|x|^{p} \\
= & : a|x|^{p},
\end{aligned}
$$

where $a$ is a positive constant since $c_{k}$ is positive. Hence, we obtain that

$$
a|x|^{p} \leq V(x, t) \leq b|x|^{p} .
$$

So condition (2) in Lemma 2 is satisfied.

On the other hand, using (V2) we have

$$
\begin{aligned}
L V(x(t), t) & \leq-\sum_{k=1}^{l} c_{k} \sigma_{k}\left|x_{k}(t)\right|^{p}+\sum_{k, h=1}^{l} c_{k} a_{k h} F_{k h}\left(x_{k}(t), x_{h}(t)\right)+\sum_{k=1}^{l} c_{k} \gamma_{k} \\
& \leq-\sigma|x(t)|^{p}+\sum_{k, h=1}^{l} c_{k} a_{k h} F_{k h}\left(x_{k}(t), x_{h}(t)\right)+\sum_{k=1}^{l} c_{k} \gamma_{k},
\end{aligned}
$$


where $\sigma=\left(\sum_{k=1}^{l} c_{k} \sigma_{k}\right)^{1-\frac{p}{2}}\left(\min _{k \in \mathbb{L}}\left\{c_{k} \sigma_{k}\right\}\right)^{\frac{p}{2}}>0$. From Lemma 1 and condition $(\mathbf{V 3})$ we observe that

$$
\sum_{k, h=1}^{l} c_{k} a_{k h} F_{k h}\left(x_{k}, x_{h}\right)=\sum_{Q \in \mathbb{Q}} W(Q) \sum_{(s, r) \in E\left(C_{Q}\right)} F_{r s}\left(x_{r}, x_{s}\right) \leq 0,
$$

which implies that

$$
L V(x(t), t) \leq-\sigma|x(t)|^{p}+\sum_{k=1}^{l} c_{k} \gamma_{k}
$$

From this it follows that $L V \rightarrow-\infty$ as $|x| \rightarrow \infty$. Then the conclusion of Theorem 1 follows from Lemma 2.

Remark 1. As stated in the Introduction, the issue of global stability of coupled systems on networks is an interesting research topic and many results have been reported. However, periodicity has not been studied for the stochastic coupled systems so far. Based on the graph theory and Lyapunov method, we firstly develop a systematic method to obtain the existence of periodic solutions for stochastic coupled systems on networks.

Remark 2. Theorem 1 shows that it is important to construct a suitable global Lyapunov function $V(x)$ for the coupled systems on networks using individual $V_{k}\left(x_{k}\right)$. In many applied fields, Lyapunov functions $V_{k}\left(x_{k}\right), k \in \mathbb{L}$ can be chosen as the well-known Lyapunov functions for individual vertex systems. We can see that the strong connectedness of $(\mathcal{G}, A)$ plays an important role in the construction of the global Lyapunov functions.

\section{The existence and global asymptotic stability of periodic solutions to a type of coupled system on networks}

In this section, to demonstrate the correctness and applicability of our main result, we consider the following stochastic coupled system on networks.

$$
\begin{aligned}
\mathrm{d} x_{k}(t)= & {\left[A(t) x_{k}(t)+f_{k}\left(t, x_{k}(t)\right)+I_{k}(t)+\sum_{h=1}^{l} H_{k h}\left(t, x_{h}(t)\right)\right] \mathrm{d} t+g_{k}\left(t, x_{k}(t)\right) \mathrm{d} B_{k}(t)+\sum_{h=1}^{l} \sigma_{k h}\left(t, x_{h}(t)\right) \mathrm{d} B_{h}(t), } \\
& 1 \leq k \leq l, t \geq 0 .
\end{aligned}
$$

where $x_{k}(t)=\left(x_{k 1}(t), x_{k 2}(t), \cdots, x_{k m}(t)\right)^{T}$ represents the state of the $k$-th subsystem, $A(t) \in C\left(\mathbb{R}^{+}, \mathbb{R}^{m \times m}\right), I_{k}(t) \in$ $C\left(\mathbb{R}^{+}, \mathbb{R}^{m}\right)$, functions $f_{k}=\left(f_{k 1}, f_{k 2}, \cdots, f_{k m}\right)^{T}: \mathbb{R}^{+} \times \mathbb{R}^{m} \rightarrow \mathbb{R}^{m}, g_{k}=\left(g_{k 1}, g_{k 2}, \cdots, g_{k m}\right)^{T}: \mathbb{R}^{+} \times \mathbb{R}^{m} \rightarrow \mathbb{R}^{m}$ are continuous. $H_{k h}\left(t, x_{h}(t)\right)$ and $\sigma_{k h}\left(t, x_{h}(t)\right)$ represent the influence of the $h$-th subsystem on the $k$-th subsystem for the drift coefficient and the diffusion coefficient, respectively. Here $H_{k k}=\sigma_{k k}=0$ and $H_{k h}=\sigma_{k h}=0$ if and only if there exists no influence from $h$-th subsystem to $k$-th subsystem.

For convenience, we will introduce the following assumptions.

(A1) For any $k \in \mathbb{L}, A(t), f_{k}(t, x), H_{k h}(t, x), I_{k}(t), g_{k}(t, x)$ and $\sigma_{k h}(t, x)$ are continuous $T$-periodic functions in $t$.

(A2) Functions $f_{k}(t, x), H_{k h}(t, x), g_{k}(t, x)$ and $\sigma_{k h}(t, x)$ satisfy the Lipschitz condition, that is, there exist $\alpha_{k}>0$, $\beta_{k}>0, a_{k h} \geq 0$ such that

$$
\begin{aligned}
& \left|f_{k}(t, u)-f_{k}(t, v)\right| \leq \alpha_{k}|u-v|, \\
& \left|g_{k}(t, u)-g_{k}(t, v)\right| \leq \beta_{k}|u-v|, \\
& \left|H_{k h}(t, u)-H_{k h}(t, v)\right| \leq a_{k h}|u-v|, \\
& \left|\left(\sigma_{k h}(t, u)-\sigma_{k h}(t, v)\right)\left(\sigma_{k h}(t, u)-\sigma_{k h}(t, v)\right)^{T}\right| \leq a_{k h}|u-v|^{2}, \\
& \forall u, v \in \mathbb{R}^{m}, \quad \forall k=1,2, \cdots, l, \quad t \geq 0 .
\end{aligned}
$$


Without loss of generality, we also assume that $f_{k}(t, 0)=H_{k h}(t, 0)=g_{k}(t, 0)=\sigma_{k h}(t, 0)=0$.

Remark 3. It follows from conditions (A1) and (A2) that the coefficients of system (6) satisfy the Lipschitz condition and linear growth condition, so there exists a unique global solution of system (6).

Theorem 2. Assume that conditions (A1) and (A2) hold. Furthermore, suppose that the following conditions hold.

(A3) $\lambda_{\max }\left(\frac{A(t)+A^{T}(t)}{2}\right) \leq-\rho, \forall t \in \mathbb{R}^{+}$, where $\lambda_{\max }(A)$ is the maximum eigenvalue of matrix $A$.

(A4) Digraph $(\mathcal{G}, A)\left(A=\left(a_{k h}\right)_{l \times l}\right)$ is strongly connected.

(A5) For any $k \in \mathbb{L}, \rho>\alpha_{k}+\frac{\beta_{k}^{2}}{2}+\frac{3}{2} \sum_{h=1}^{l} a_{k h}$.

Then system (6) has a T-periodic solution.

Proof. Define a Lyapunov function

$$
V_{k}\left(x_{k}\right)=\left|x_{k}\right|^{2},
$$

which satisfies condition (V1) of Theorem 1. From the condition (A5), we can deduce that there exists a sufficient small constant $\varepsilon>0$ such that $\rho>\alpha_{k}+\frac{\beta_{k}^{2}}{2}+\frac{\varepsilon^{2}}{2}+\frac{3}{2} \sum_{h=1}^{l} a_{k h}$. Then by the conditions (A1)-(A3), we get

$$
\begin{aligned}
L V_{k} & =2 x_{k}^{T}(t)\left[A(t) x_{k}(t)+f_{k}\left(t, x_{k}(t)\right)+I_{k}(t)+\sum_{h=1}^{l} H_{k h}\left(t, x_{h}(t)\right)\right]+\left|g_{k}\left(t, x_{k}(t)\right)\right|^{2}+\sum_{h=1}^{l}\left|\sigma_{k h}\left(t, x_{h}(t)\right)\right|^{2} \\
& =2 x_{k}^{T}(t) \frac{A(t)+A^{T}(t)}{2} x_{k}(t)+2 \alpha_{k}\left|x_{k}(t)\right|^{2}+2 I_{k}^{u}\left|x_{k}(t)\right|+2 \sum_{h=1}^{l} a_{k h}\left|x_{k}(t)\right|\left|x_{h}(t)\right|+\beta_{k}^{2}\left|x_{k}(t)\right|^{2}+\sum_{h=1}^{l} a_{k h}\left|x_{h}(t)\right|^{2} \\
& \leq-2 \rho\left|x_{k}(t)\right|^{2}+2 \alpha_{k}\left|x_{k}(t)\right|^{2}+\varepsilon^{2}\left|x_{k}(t)\right|^{2}+\frac{\left(I_{k}^{u}\right)^{2}}{\varepsilon^{2}}+\sum_{h=1}^{l} a_{k h}\left(\left|x_{k}(t)\right|^{2}+\left|x_{h}(t)\right|^{2}\right)+\beta_{k}^{2}\left|x_{k}(t)\right|^{2}+\sum_{h=1}^{l} a_{k h}\left|x_{h}(t)\right|^{2} \\
& =-2\left(\rho-\alpha_{k}-\frac{\beta_{k}^{2}}{2}-\frac{\varepsilon^{2}}{2}-\frac{3}{2} \sum_{h=1}^{l} a_{k h}\right)\left|x_{k}(t)\right|^{2}+\frac{\left(I_{k}^{u}\right)^{2}}{\varepsilon^{2}}+\sum_{h=1}^{l} a_{k h} F_{k h}\left(x_{k}(t), x_{h}(t)\right) .
\end{aligned}
$$

where $F_{k h}\left(x_{k}, x_{h}\right)=2\left(\left|x_{h}\right|^{2}-\left|x_{k}\right|^{2}\right)$. In the meanwhile, we have

$$
\sum_{(s, r) \in E(C)} F_{r s}\left(x_{r}(t), x_{s}(t)\right)=\sum_{(s, r) \in E(\mathcal{C})} 2\left(\left|x_{s}(t)\right|^{2}-\left|x_{r}(t)\right|^{2}\right)=0,
$$

where $C$ is any directed cycle of the weighted digraph $(\mathcal{G}, A)$. Hence, assumptions (V2) and (V3) have been verified. Then by Theorem 1, system (6) has a $T$-periodic solution. The proof is complete.

Theorem 3. Assume that all the conditions of Theorem 2 hold. Then the periodic solution of (6) is globally asymptotically stable.

Proof. Let $x^{*}(t)=\left(x_{1}^{*}(t), \cdots, x_{l}^{*}(t)\right)^{T}$ be a $T$-periodic solution of system (6). Let $u_{k}(t)=x_{k}(t)-x_{k}^{*}(t)$. Then we have

$$
\begin{aligned}
\mathrm{d} u_{k}(t)= & {\left[A(t) u_{k}(t)+f_{k}\left(t, x_{k}(t)\right)-f_{k}\left(t, x_{k}^{*}(t)\right)+\sum_{h=1}^{l}\left(H_{k h}\left(t, x_{h}(t)\right)-H_{k h}\left(t, x_{h}^{*}(t)\right)\right)\right] \mathrm{d} t } \\
& +\left(g_{k}\left(t, x_{k}(t)\right)-g_{k}\left(t, x_{k}^{*}(t)\right)\right) \mathrm{d} B_{k}(t)+\sum_{h=1}^{l}\left(\sigma_{k h}\left(t, x_{h}(t)\right)-\sigma_{k h}\left(t, x_{h}^{*}(t)\right)\right) \mathrm{d} B_{h}(t) .
\end{aligned}
$$


Denote $V_{k}\left(u_{k}\right)=\left|u_{k}\right|^{2}$. By the conditions (A1)-(A3), we get

$$
\begin{aligned}
L V_{k}\left(u_{k}(t)\right)= & 2 u_{k}^{T}(t) A(t) u_{k}(t)+2 u_{k}^{T}(t)\left(f_{k}\left(t, x_{k}(t)\right)-f_{k}\left(t, x_{k}^{*}(t)\right)\right)+2 \sum_{h=1}^{l} u_{k}^{T}(t)\left(H_{k h}\left(t, x_{h}(t)\right)-H_{k h}\left(t, x_{h}^{*}(t)\right)\right) \\
& +\left|g_{k}\left(t, x_{k}(t)\right)-g_{k}\left(t, x_{k}^{*}(t)\right)\right|^{2}+\sum_{h=1}^{l}\left|\sigma_{k h}\left(t, x_{h}(t)\right)-\sigma_{k h}\left(t, x_{h}^{*}(t)\right)\right|^{2} \\
= & 2 u_{k}^{T}(t) \frac{A(t)+A^{T}(t)}{2} u_{k}(t)+2 \alpha_{k}\left|u_{k}(t)\right|^{2}+2 \sum_{h=1}^{l} a_{k h}\left|u_{k}(t)\right|\left|u_{h}(t)\right|+\beta_{k}^{2}\left|u_{k}(t)\right|^{2}+\sum_{h=1}^{l} a_{k h}\left|u_{h}(t)\right|^{2} \\
\leq & -2\left(\rho-\alpha_{k}-\frac{\beta_{k}^{2}}{2}-\frac{3}{2} \sum_{h=1}^{l} a_{k h}\right)\left|u_{k}(t)\right|^{2}+\sum_{h=1}^{l} a_{k h} F_{k h}\left(u_{k}(t), u_{h}(t)\right),
\end{aligned}
$$

where $F_{k h}\left(u_{k}(t), u_{h}(t)\right)=2\left(\left|u_{h}(t)\right|^{2}-\left|u_{k}(t)\right|^{2}\right)$. Set $V(u)=\sum_{k=1}^{l} c_{k} V_{k}\left(u_{k}\right), u=\left(u_{1}, u_{2}, \ldots, u_{l}\right)^{T}, c_{k}$ is the cofactor of the $k$-th diagonal element of Laplacian matrix of $\left(\mathcal{G},\left(a_{k h}\right)_{l \times l}\right)$. From $(9)$ and Lemma 1 it follows that

$$
\begin{aligned}
L V(u(t)) & \leq-2 \min _{k \in \mathbb{L}}\left\{\left(\rho-\alpha_{k}-\frac{\beta_{k}^{2}}{2}-\frac{3}{2} \sum_{h=1}^{l} a_{k h}\right) c_{k}\right\} \sum_{k=1}^{l}\left|u_{k}(t)\right|^{2}+\sum_{k, h=1}^{l} c_{k} a_{k h} F_{k h}\left(u_{k}(t), u_{h}(t)\right) \\
& \leq-2 \min _{k \in \mathbb{L}}\left\{\left(\rho-\alpha_{k}-\frac{\beta_{k}^{2}}{2}-\frac{3}{2} \sum_{h=1}^{l} a_{k h}\right) c_{k}\right\} \sum_{k=1}^{l}\left|u_{k}(t)\right|^{2} \\
& \leq 0 .
\end{aligned}
$$

Hence from [31] we deduce that the zero solution of (8) is globally asymptotically stable, that is, the periodic solution $x^{*}(t)$ of $(6)$ is globally asymptotically stable. This completes the proof.

Remark 4. From the proofs of Theorems 1,2 and 3, we see that the strong connectedness of digraph $(\mathcal{G}, A)$ implies that $c_{k}>0$ which is need. Hence the theoretical results can be extend to the case where all the cofactors of the diagonal elements of Laplacian matrix of $(\mathcal{G}, A)$ are positive.

Remark 5. In paper [18], Li and Shuai investigated the global-stability problem of equilibria for coupled systems of differential equations on networks by using graph theory and Lyapunov functions. In our paper, using the methods developed by Li and Shuai, combining Has'minskii theory of periodic solution, we study the periodic solutions to the stochastic coupled systems on networks in periodic environments. Moreover, the global asymptotic stability of the periodic solution to a class of stochastic coupled system on networks is also investigated. Hence, the main advantage in this paper is that we generalize the results and methods in [18] to the case of stochastic coupled systems in periodic environments. 


\section{Example and numerical simulations}

In this section, to support our analytical results, we give a numerical example. Consider the following coupled system:

$$
\begin{aligned}
\mathrm{d} x_{k 1}(t)= & {\left[-2.5 x_{k 1}(t)+\mu_{k}(t) \sin \left(x_{k 1}(t)\right)+v_{k}(t) x_{k 2}(t)+0.1 k+\sin t+\sum_{h=1}^{4} a_{k h} x_{h 1}(t)\right] \mathrm{d} t } \\
& +\left[\bar{\mu}_{k}(t) x_{k 1}(t)+\bar{v}_{k}(t) \sin \left(x_{k 2}(t)\right)\right] \mathrm{d} B_{k}(t)+\sum_{h=1}^{4} \sqrt{a_{k h}} \sin \left(x_{h 1}(t)\right) \mathrm{d} B_{h}(t), \\
\mathrm{d} x_{k 2}(t)= & {\left[-2.5 x_{k 2}(t)+\mu_{k}(t) \sin \left(x_{k 2}(t)\right)+v_{k}(t) x_{k 1}(t)+0.1 k+\cos t+\sum_{h=1}^{4} a_{k h} x_{h 2}(t)\right] \mathrm{d} t } \\
& +\left[\bar{\mu}_{k}(t) x_{k 2}(t)+\bar{v}_{k}(t) \sin \left(x_{k 1}(t)\right)\right] \mathrm{d} B_{k}(t)+\sum_{h=1}^{4} \sqrt{a_{k h}} \sin \left(x_{h 2}(t)\right) \mathrm{d} B_{h}(t) .
\end{aligned}
$$

where $x_{k}(t)=\left(x_{k 1}(t), x_{k 2}(t)\right)^{T}, k=1,2,3,4$. Let

$$
\begin{gathered}
A(t)=\left(\begin{array}{cc}
-2.5 & 0 \\
0 & -2.5
\end{array}\right), \\
f_{k}\left(t, x_{k}(t)\right)=\left(\begin{array}{c}
\mu_{k}(t) \sin \left(x_{k 1}(t)\right)+v_{k}(t) x_{k 2}(t) \\
\mu_{k}(t) \sin \left(x_{k 2}(t)\right)+v_{k}(t) x_{k 1}(t)
\end{array}\right), \\
g_{k}\left(t, x_{k}(t)\right)=\left(\begin{array}{c}
\bar{\mu}_{k}(t) x_{k 1}(t)+\bar{v}_{k}(t) \sin \left(x_{k 2}(t)\right) \\
\bar{\mu}_{k}(t) x_{k 2}(t)+\bar{v}_{k}(t) \sin \left(x_{k 1}(t)\right)
\end{array}\right), \\
H_{k h}\left(t, x_{h}(t)\right)=\left(a_{k h} x_{h 1}(t), a_{k h} x_{h 2}(t)\right)^{T}, \quad \sigma_{k h}\left(t, x_{h}(t)\right)=\left(\sqrt{a_{k h}} \sin \left(x_{h 1}(t)\right), \sqrt{a_{k h}} \sin \left(x_{h 2}(t)\right)\right)^{T}, \\
I_{k}(t)=(0.1 \times k+\sin t, 0.1 \times k+\cos t)^{T},
\end{gathered}
$$

where

$$
\mu_{k}(t)=\frac{1+\sin t}{2 k}, \quad v_{k}(t)=\frac{1+\cos ^{2} t}{2 k}, \quad \bar{\mu}_{k}(t)=\frac{1+\cos ^{2} t}{20 k}, \quad \bar{v}_{k}(t)=\frac{1+\sin t}{20 k},
$$

and the coupling graph of system (11) is assumed that

$$
\left(a_{k h}\right)_{4 \times 4}=\left(\begin{array}{cccc}
0 & 0.2 & 0.3 & 0.4 \\
0.2 & 0 & 0.5 & 0.2 \\
0.3 & 0.5 & 0 & 0.1 \\
0.4 & 0.2 & 0.1 & 0
\end{array}\right) .
$$

Then we have

$$
\lambda_{\max }\left(\frac{A(t)+A^{T}(t)}{2}\right)=-2.5, \quad \max _{k \in \mathbb{L}} \sum_{h=1}^{l} a_{k h}=0.9
$$

and

$$
\begin{gathered}
\left|f_{k}(t, u)-f_{k}(t, v)\right| \leq|u-v| \\
\left|g_{k}(t, u)-g_{k}(t, v)\right| \leq 0.1|u-v|,
\end{gathered}
$$




$$
\begin{gathered}
\left|H_{k h}(t, u)-H_{k h}(t, v)\right| \leq a_{k h}|u-v|, \\
\left|\left(\sigma_{k h}(t, u)-\sigma_{k h}(t, v)\right)\left(\sigma_{k h}(t, u)-\sigma_{k h}(t, v)\right)^{T}\right| \leq a_{k h}|u-v|^{2} .
\end{gathered}
$$

So the conditions of Theorems 2 and 3 are all satisfied. Hence system (11) has a unique global asymptotic stable $2 \pi$-periodic solution, which is shown in Figures 2, 3, 4 and 5 with initial values $\left(x_{11}(0), x_{12}(0)\right)=$ $(-2,-3),(-1,-2),(-2.5,-1.5),\left(x_{21}(0), x_{22}(0)\right)=(-5,2),(-6,1),(-4,3),\left(x_{31}(0), x_{32}(0)\right)=(4,-1),(3,-2),(5,1)$ and $\left(x_{41}(0), x_{42}(0)\right)=(3,4),(2,3),(4,5)$.

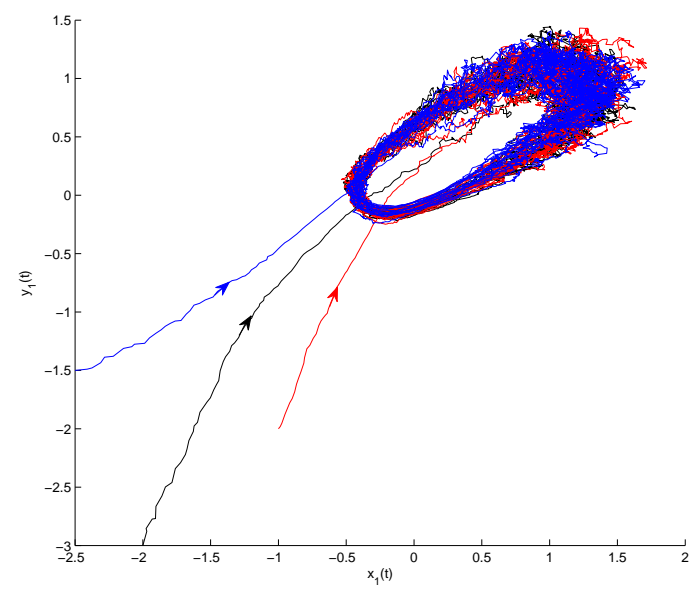

Figure 2: The phrase plane of the subsystem $x_{1}$ of system (11) with initial value $\left(x_{11}(0), x_{12}(0)\right)=(-2,-3),(-1,-2),(-2.5,-1.5)$.

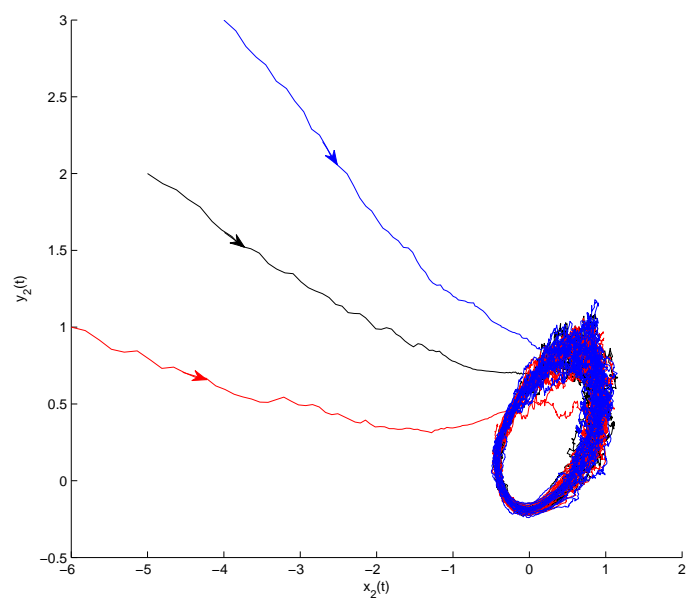

Figure 3: The phrase plane of the subsystem $x_{2}$ of system (11) with initial value $\left(x_{21}(0), x_{22}(0)\right)=(-5,2),(-6,1),(-4,3)$.

\section{Conclusion and future Directions}

In this paper, we study a class of stochastic coupled systems on networks with periodic coefficients. Using the combined method of graph theory and Lyapunov method, we develop a systematic approach that allows one to 


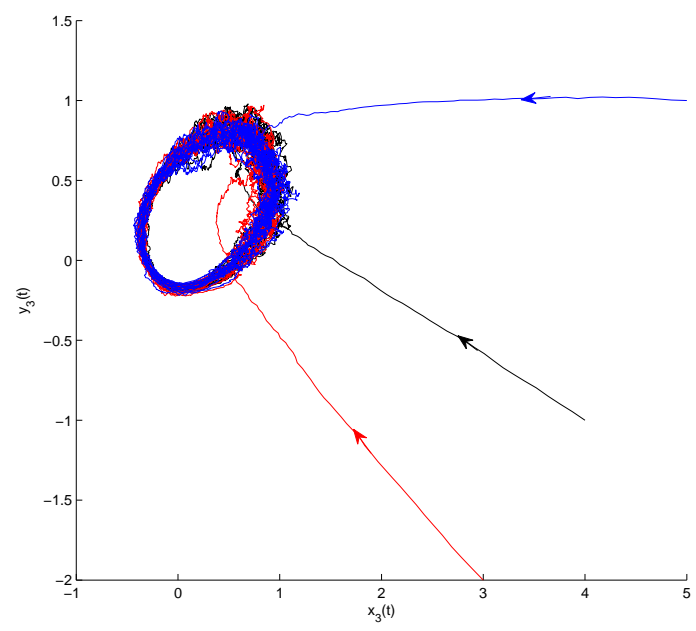

Figure 4: The phrase plane of the subsystem $x_{3}$ of system (11) with initial value $\left(x_{31}(0), x_{32}(0)\right)=(4,-1),(3,-2),(5,1)$.

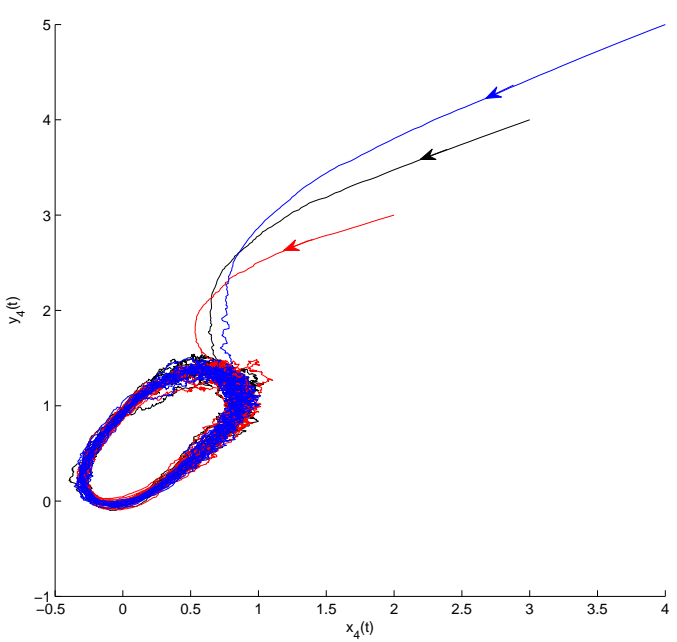

Figure 5: The phrase plane of the subsystem $x_{4}$ of system (11) with initial value $\left(x_{41}(0), x_{42}(0)\right)=(3,4),(2,3),(4,5)$. 
obtain the existence of periodic solutions for stochastic coupled systems on networks. We apply this approach to a type of stochastic coupled system and derive the sufficient conditions for the existence and global asymptotic stability of periodic solution.

We can see that in the proof of our main result, the graph theory is effective to study the coupled systems on networks. Hence, the combined method of graph theory and Lyapunov function is of decided advantage in studying the existence of periodic solutions for large-scale coupled systems.

We would like to mention that results reported here are not exhaustive. We will continue to do some researches about the existence of the periodic solution of stochastic coupled systems on networks with delays, stochastic neutral functional differential coupled system, some population systems and so on.

\section{Acknowledgements}

The authors thank the NSF of Shandong Province of China (No.ZR2014AL008), National Natural Science Foundation of P.R. China (Nos. 41406007, 11301112, 11401586), and the Fundamental Research Funds for the Central Universities of China (15CX02080A).

\section{Appendix}

For the sake of convenience, we introduce a few concepts and results concerning the graph theory and coincidence degree as follows. For more details see[32].

Graph theory. A digraph $\mathcal{G}=(U, E)$ contains a set $U=\{1,2, \cdots, l\}$ of vertices and a set $E$ of $\operatorname{arcs}(k, h)$ leading from initial vertex $k$ to terminal vertex $h$. A subgraph $\mathcal{H}$ of $\mathcal{G}$ is said to be spanning if $\mathcal{H}$ and $\mathcal{G}$ have the same vertex set. A digraph $\mathcal{G}$ is weighted if each $\operatorname{arc}(h, k)$ is assigned a positive weight $a_{k h}$. Here $a_{k h}>0$ if and only if there exists an arc from vertex $h$ to vertex $k$ in $\mathcal{G}$, and we call $A=\left(a_{k h}\right)_{l \times l}$ as the weight matrix. The weight $W(\mathcal{G})$ of $\mathcal{G}$ is the product of the weights on all its arcs.

A directed path $\mathcal{P}$ in $\mathcal{G}$ is a subgraph with distinct vertices $\left\{i_{1}, i_{2}, \cdots, i_{s}\right\}$ such that its set of arcs is $\left\{\left(i_{k}, i_{k+1}\right)\right.$ : $k=1,2, \cdots, s-1\}$. If $i_{s}=i_{1}$, we call $\mathcal{P}$ a directed cycle. A connected subgraph $\mathcal{T}$ is a tree if it contains no cycles. A tree $\mathcal{T}$ is rooted at vertex $k$, called the root, if $k$ is not a terminal vertex of any arcs, and each of the remaining vertices is a terminal vertex of exactly one arc. A subgraph $Q$ is unicyclic if it is a disjoint union of rooted trees whose roots form a directed cycle.

A digraph $\mathcal{G}$ is strongly connected if, for any pair of distinct vertices, there exists a directed path from one to the other. Denote the digraph with weight matrix $A$ as $(\mathcal{G}, A)$. The Laplacian matrix of $(\mathcal{G}, A)$ is defined as

$$
L=\left(\begin{array}{cccc}
\sum_{k \neq 1} a_{1 k} & -a_{12} & \cdots & -a_{1 l} \\
-a_{21} & \sum_{k \neq 2} a_{2 k} & \cdots & -a_{2 l} \\
\vdots & \vdots & \ddots & \vdots \\
-a_{l 1} & -a_{l 2} & \cdots & \sum_{k \neq l} a_{l k}
\end{array}\right) .
$$

A weighted digraph $(\mathcal{G}, A)$ is said to be balanced if $W(C)=W(-C)$ for all directed cycles $C$. Here, $-C$ denotes the reverse of $C$ and is constructed by reversing the direction of all $\operatorname{arcs}$ in $C$. For a unicyclic graph $Q$ with cycle $C_{Q}$, let $\tilde{Q}$ be the unicyclic graph obtained by replacing $C_{Q}$ with $-C_{Q}$. Suppose that $(\mathcal{G}, A)$ is balanced, then $W(Q)=W(\tilde{Q})$. 


\section{References}

[1] B. Øksendal, Stochastic Differential Equations: An Introduction with Applications, 6thed, SpringerVerlag, Berlin Heidelberg, 2003.

[2] L. Arnold, Stochastic Differential Equations: Theory and Applications, Wiley, New York, 1972.

[3] A. Friedman, Stochastic Differential Equations and Their Applications, Vol 2, Academic Press, New York, 1976.

[4] R.Z.Has'minskii, Stochastic Stability of Differential Equations, Sijthoff and Noordhoff, Alphen, 1981.

[5] X. Mao, C. Yuan, Stochastic Differential Equations with Markovian Switching, Imperial College Press, London, 2006.

[6] X. Mao, G. Marion, E. Renshaw, Environmental Brownian noise suppresses explosions in population dynamics, Stoch. Proc. Appl. 97 (2002) 95-110.

[7] B. Zhang, K.Gopalsamy, On the periodic solution of n-dimensional stochastic population models, Stoch. Anal. Appl. 18 (2000) 323-331.

[8] D. Xu, Y. Huang, Z. Yang, Existence theorems for periodic Markov process and stochastic functional differential equations, Discrete. Contin. Dyn. Syst. 24 (2009) 1005-1023.

[9] D. Li, D. Xu, Periodic solutions of stochastic delay differential equations and applications to Logisitc equation and neural networks, J. Korean Math. Soc. 50 (2013) 1165-1181.

[10] D. Li, X. Wang, D. Xu, Existence and global p-exponential stability of periodic solution for impulsive stochastic neural networks with delays, Nonlinear Anal. Hybrid Syst. 6 (2012) 847-858.

[11] C. Bishop, Neural Networks for Pattern Recognition, Oxford University Press, Oxford, 1995.

[12] R.M. May, Stability and Complexity in Model Ecosystems, Princeton University Press, Princeton, 2001.

[13] H.R. Thieme, Mathematics in Population Biology, Princeton University Press, Princeton, 2003.

[14] R.V. Sole, J. Bascompte, Self-Organization in Complex Ecosystems, Princeton University Press, Princeton, 2006.

[15] Y. Dai, Y. Cai, X. Xu, Synchronization criteria for complex dynamical networks with neutral-type coupling delay, Phys. A 387 (2008) 4673-4682.

[16] H. Liu, J. Chen, J. Lu, M. Cao, Generalized synchronization in complex dynamical networks via adaptive couplings, Phys. A 389 (2010) 1759-1770.

[17] B. Shen, Z. Wang, X. Liu, Bounded H-infinity synchronization and state estimation for discrete timevarying stochastic complex networks over a finite-horizon, IEEE Trans. Neural Netw. 22 (2011) 145-157.

[18] M.Y. Li, Z. Shuai, Global-stability problem for coupled systems of differential equations on networks, J. Differ. Equ. 248 (2010) 1-20. 
[19] H. Su, W. Li, K. Wang, Global stability analysis of discrete-time coupled systems on networks and its applications, Chaos 22 (2012) 033135.

[20] W. Li, H. Su, K. Wang, Global stability analysis for stochastic coupled systems on networks, Automatica 47 (2011) 215-220.

[21] X. Zhang, W. Li, K.Wang, The existence of periodic solutions for coupled systems on networks with time delays, Neurocomputing 152 (2015) 287-293.

[22] X. Zhang,W. Li, K.Wang, The existence and global exponential stability of periodic solution for a neutral coupled system on networks with delays, Appl. Math. Comput. 264 (2015) 208-217.

[23] J. Suo, J. Sun, Y. Zhang, Stability analysis for impulsive coupled systems on networks, Neurocomputing 99 (2013) 172-177.

[24] H. Guo, M.Y. Li, Z. Shuai, Global dynamics of a general class of multistage models for infectious diseases, SIAM J. Appl. Math. 72 (2012) 261-279.

[25] H. Shu, D. Fan, J. Wei, Global stability of multi-group SEIR epidemic models with distributed delays and nonlinear transmission, Nonlinear Anal. RWA. 13 (2012) 1581-1592.

[26] L. Wen, J. Zhong, Global asymptotic stability and a property of the SIS model on bipartite networks, Nonlinear Anal. RWA. 13 (2012) 967-976.

[27] T. Kuniya, Global stability analysis with a discretization approach for an age-structured multigroup SIR epidemic model, Nonlinear Anal. RAW. 12 (2011) 2640-2655.

[28] H. Guo, M.Y. Li, Z. Shuai, A graph-theoretic approach to the method of global Lyapunov functions, Proc. Amer. Math. Soc. 136 (2008) 2793-2802.

[29] W. Li, L. Pang, H. Su, K. Wang, Global stability for discrete Cohen-Grossberg neural networks with finite and infinite delays, Appl. Math. Lett. 25 (2012) 2246-2251.

[30] T.C. Gard, Introduction to Stochastic Differential Equations, Marcel Dekker, New York, 1987.

[31] X. Mao, A note on the LaSalle-type theorems for stochastic differential delay equations, J. Math. Anal.Appl. 268 (2002) 125-142.

[32] D.B. West, Introduction to Graph Theory, Prentice Hall, Upper Saddle River, 1996. 